\title{
Financial Time Series Forecasting Based on Characterized Candlestick and the Support Vector Classification with Cooperative Coevolution
}

\author{
Jiang Zhipeng1, Luo Chao ${ }^{1,2,3^{*}}$ \\ ${ }^{1}$ School of Information Science and Engineering, Shandong Normal University, Jinan 250014, China. \\ ${ }^{2}$ Shandong Provincial Key Laboratory for Novel Distributed Computer Software Technology, Jinan 250014, \\ China. \\ 3Institute of Data Science and Technology, Shandong Normal University, Jinan 250014, China. \\ * Corresponding author. Email: 13066033183@163.com \\ Manuscript submitted December 10, 2018; accepted February 8, 2019. \\ doi: 10.17706/jcp.14.3.195-209
}

\begin{abstract}
The fluctuations in prices in financial derivative market is an important indicator to a country or region's economic development, hence, predication of prices of financial derivatives is the research focus at present. However, the fluctuations in prices in financial market has high degree of nonlinearity, the traditional mathematical model has limitation to some extent, which influences the accuracy of prediction. This paper uses characterized Candlestick technique to implement noise removal processing on financial data and combines cooperative coevolution algorithms (CCEA) and support vector machine (SVM) to acquire the accuracy of classified prediction. After the noise removal processing made by characterized Candlestick technique, the core features of financial time series have been extracted, the randomness of financial data has been reduced, and the complexity of modeling has been simplified. The combination of CCEA and SVM has lifted the parameter optimization performance, acquired higher accuracy of classified prediction, and fit the solution of complex models. According to the computer simulation experiment, real stock data is used to verify the algorithm above, which has proved the high accuracy of prediction of this algorithm and the universality of this model.
\end{abstract}

Key words: Classified prediction, support vector machine, characterized candlestick, cooperative coevolution algorithms.

\section{Introduction}

The financial derivative market is influenced by politics, economy and participant's emotions. The system is highly nonlinear and has the characteristics of local randomness and global trend. Therefore, it is of great practical significance to track the trend of market movements and build the appropriate prediction model on this basis to effectively reduce the investment risk and raise the investment reward. With the continuous development of financial markets and improve, more and more high to the requirement of investors, early investors rely on their own subjective experience and judgment to make decisions to choose the speculation in the existing market conditions has been difficult to obtain a higher prediction accuracy. At the same time, with the rapid development of artificial intelligence technology represented by computer and machine learning, market prediction research based on financial data has become a hot spot in recent years.

SVM [1] is a classification technique proposed by Vapnik led by the experimental group based on 
statistical learning theory in 1995 [2]-[4], which is widely used in images [5], voice [6], natural language [7], [8] and other fields. Among them, SVM classification technology is also concerned in the field of financial market forecasting. Kyong-jae et al. used SVM and reverse propagation neural network to predict financial time series, which proved that SVM has the potential to deal with the prediction of financial time series [9]. Wang L et al. improved the performance of the prediction model by using SVM to predict the financial time series by adding two-step kernel function [10]. He H and Starzyk J A combined SVM with self-organizing mapping to improve prediction classification accuracy [11]. Chi-jie Lu et al. used independent partial analysis method and SVM to predict financial time series, which improved the overall performance [12].

Candlestick graph [13] this chart source in Japan's tokugawa era, was Japanese rice market traders used to record the market price fluctuations and forecast, because of its good at capturing the time series model changes after core characteristics and was introduced into the stock market and futures market. At present, this kind of chart analysis is particularly popular in the asia-pacific region. The Candlestick is drawn at the opening, the highest, the lowest and the closing price of each analytical cycle. To draw the daily candlestick as an example, first determine the price of the opening and closing prices, and the parts between them are drawn as rectangular entities. If the closing price is higher than the opening price, the candlestick is called the positive line and is represented by a hollow entity. Instead, it is called a negative line with a black entity or a white entity. Characteristic Candlestick refers to the combination form of single root, two or multi-root Candlesticks with the same characteristics, and its occurrence tends to reflect a certain pattern of subsequent financial price movements. Financial derivatives market is affected by various factors, make the financial data, especially financial time series data of a large number of noise [14], cause the stability characteristics of time series, and greatly influenced on the analysis and processing. In view of the above situation, it is particularly important to work on the de-noising of financial time series. However, the financial time series itself has the characteristics of non-stationarity, non-linearity and high SNR, and the traditional de-noising method often has many defects. In this paper, the characteristic Candlestick based on financial time series data is used as the training data of classifier.

The traditional evolutionary algorithm has achieved good results in solving the problem of low dimension, and when the number of variables is small, the optimal solution can be found quickly. However, when the problem scale increases, the difficulty of solving the problem also increases dramatically, and many classical evolutionary algorithms lose their performance and performance in the low dimension. This is the so-called Curse of Dimensionality. Traditional classical algorithms have limitations in solving high-dimensional problems. Many optimization problems in practical engineering application are large scale, and there may be complex correlation between variables, so it is urgent to need effective and efficient large-scale optimization algorithm. Cooperative collaboration is one of the most effective strategies for improving the ability of evolution algorithm to solve high-dimensional problem [15]-[20].

Cooperative co-evolution algorithm by constructing two or more populations, to establish the relationship between them, multiple populations through interaction to improve their performance, to adapt to the dynamic evolution of complex system environment, to achieve the purpose of population optimization [21]-[24]. Compared with the co-evolution algorithm, the intelligent evolution of the traditional algorithm only USES the evolution model based on individual fitness, not considering the complex relationship between the evolution of the environment and the individual and the influence of this kind of connection to individual evolution [25]-[27]. Liable to occur as a result, the application of immature defects such as slow convergence and convergence speed. And cooperative co-evolution can make full use of the evolution of a part of the individual groups guiding role, reduce the amount of calculation, quicken the speed of convergence, and cut into local optimal value [28]-[30]. 


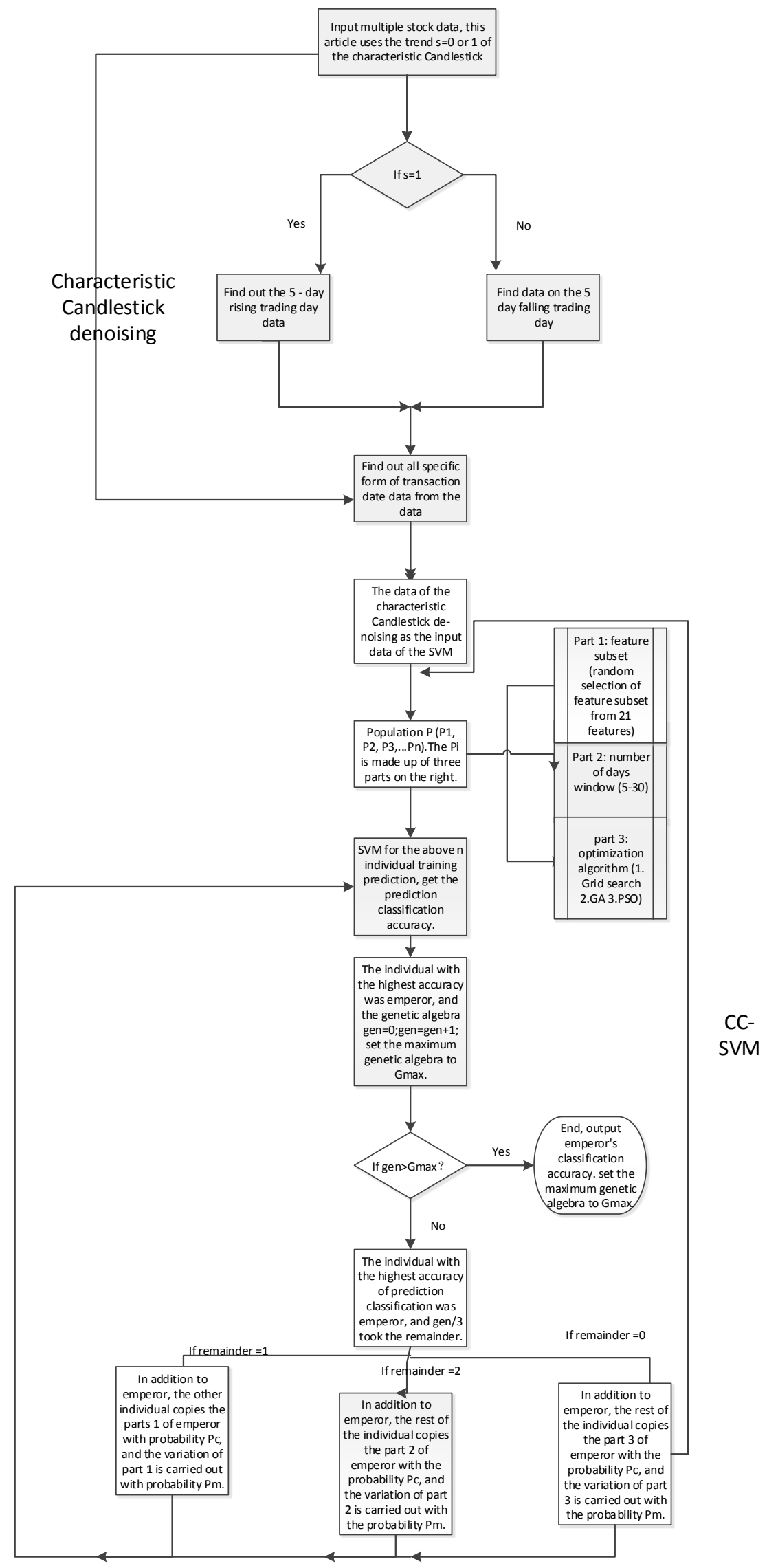

Fig. 1. Classification prediction (SVM) model graph based on feature candlestick and cooperative coevolution. 
The main problem of this paper can be summarized as follows: on the basis of processing the characterized candlestick of financial data, and then combining the cooperative co-evolution algorithm with SVM, the prediction of financial time series is studied. Firstly, based on the candlestick of financial time series, the collection of characterized candlesticks with single root, double root and multiple roots is selected. Secondly, a financial time series prediction model is constructed based on the optimization framework constructed by cooperative collaborative evolution algorithm. The optimization framework mainly consists of three parts: feature subset, window length, optimization algorithm. Feature selection and Window length together determine the data set. The dataset has an impact on SVM optimization parameters $\mathrm{c}$ and g.It is simply not enough to optimize a single component individually without considering the interaction between them. However, all three problems are judged by the final classification accuracy as the only criterion. It is precisely because these three parts are closely related, this paper uses the cooperative coevolution method based on genetic algorithm (CCGA) to optimize the above three components. The validity of the proposed algorithm is verified by real stock data.

\section{Model}

The model is shown in Fig. 1, which consists of two parts. The first part denotes the characterized candlestick, and the second part is CC-SVM.

\subsection{Methods of De-noising}

Traditional methods of de-noising financial time series are mainly moving average method [31], traditional filtering method [32], Kalman filtering method [33] and wiener filtering method [34]. As a kind of simple moving average method of data smoothing techniques, this method is very rough, while de-noising, a lot of useful information was also removed, so it is only for simple analysis of the data, not suitable for the deep analysis of the data. Traditional filtering requires that the spectrum of useful signals and noise be separated from each other. But for financial time series, such as stock price time series and its volatility yield sequence are bigger, the spectrum is wide, and the useful signal and noise spectrum overlap is serious, the traditional method is difficult to achieve effective separation of signal-to-noise. Kalman filter needs to know the motion of the system to establish an accurate equation of state. But the financial time series is a non-stationary and nonlinear time series, it is difficult to use a certain equation to describe the state and behavior, therefore this method is used to de-noising of financial time series is the inherent difficulty. Wiener filter requires prior knowledge of noise and useful signals such as their autocorrelation function and power spectral density. Because these prior knowledge is difficult to be obtained or oversimplified in practice, the optimal wiener filter is often not required. The complexity, variability and non-linearity of financial time series increase the difficulty of forecasting. The particularity of time series prediction has the limitation of traditional de-noising method.

Technical analysis refers to financial derivatives prices rise and fall of intuitive behavior as the main research object, to predict price movements form and trend for the main purpose, from the candlestick chart and technical indexes of stock price changes, analyze the law of stock market volatility method combined. Technical analysis to predict financial time series, often appears in a certain feature of candlestick as the basis of market trends, such as in the downward trend of Inverted hammer, the herald the end of the downward trend. The appearance of Hanging man in the rising trend indicates the end of the upward trend. Therefore, as shown in Fig. 1, the characterized candlestick de-noising part, taking out the same characterized candlestick in the same trend as the training data of classifier, is also the de-noising method of this paper. This characterized candlestick de-noising method originates from the traditional technical index analysis.

The traditional de-noising method considers the singular value as noise. In the financial time series, the 
occurrence of singular value is usually caused by sudden events, which usually indicates the end of trend or the enhancement of trend. Therefore, the singular value is not noise, but is research focus. The characterized candlestick fully embodies the value of the singular value. Due to the particularity of financial time series prediction, traditional de-noising method does not apply. Compared with traditional de-noising methods, the characteristics of candlestick de-noising method not only reduces the amount of data, reduce the complexity of the classified prediction, and improve the classification accuracy to a great extent, it will be in the back of the validating experiment part.

\subsection{The Use of Cooperative Coevolution Algorithm in SVM (CC-SVM)}

The idea of collaborative evolution using biological coevolution. By constructing multiple species and establishing the relationship between them, multiple species can improve their performance to achieve the goal of population optimization. The individuals of A-P in Fig. 2 are both useful to the final result, Z, and interact with each other. In this case, it's just A-P looking for their optimal solution, and obviously you can't get the optimal solution for Z. Accordingly, it presents the method of cooperative coevolution, cooperative coevolution is defined like this: by constructing multiple populations and to establish the relationship between them, multiple population to improve their performance by interaction, to adapt to the dynamic evolution of complex system environment, to achieve the goal of population optimization. The relationship between all individuals of A-P is established to adapt to the complex dynamic evolution environment and obtain a better solution of $\mathrm{Z}$.

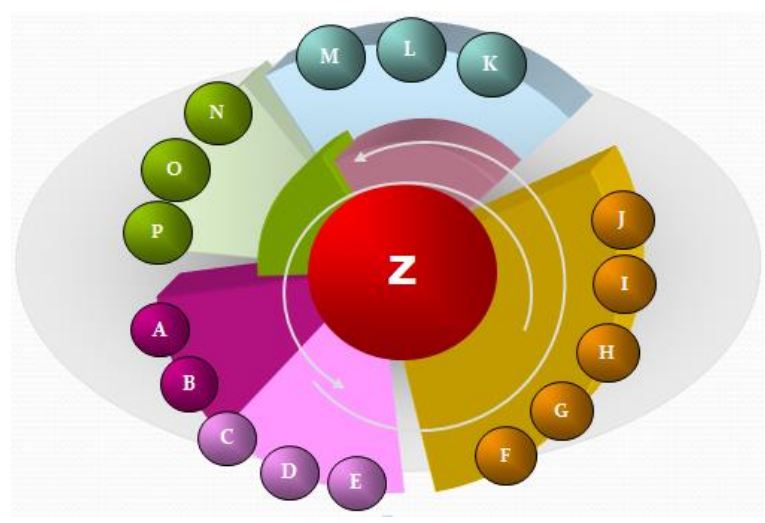

Fig. 2. Example diagram of cooperative collaborative evolution algorithm.

As shown in Fig. 1, the cooperative coevolution and support vector machine section contains feature subset, window length, optimization algorithm. Feature subset selection, from the following characteristics of DIF, DEA, MACD, DMA, AMA, PSY, CLOSE, D, RSI, OPEN, HIGH, MA5, MA10, VOLUME, K, LOW, UPPERLINE, MIDDLELINE, LOWLINE, CCI, j feature subset is selected. window length, randomly select between 5 days and 30 days. Optimization algorithm, random selection of values between 1 and 3, 1 represents grid search, 2 represents GA algorithm, and 3 represents particle swarm algorithm. In this paper, the cooperative collaborative evolutionary algorithm (CCGA) based on genetic algorithm is used to optimize these three problems. Here's how:

Initialization: genetic algebra gen $=0$, The feature subset, window length and optimization algorithm are regarded as three components. Three components of 50 individuals are randomly selected. For example, the feature set randomly selected high, low, open, close, and the number of days randomly selected 5, the optimization algorithm randomly selected GA genetic algorithm. After the training of SVM, the classification accuracy of this individual was obtained, and the other individuals got three parts randomly to get the accuracy. 
50 individuals get their respective SVM classification accuracy, choose the highest classification accuracy of the individual as emperor, gen $=$ gen +1 , if gen $/ 3$ of the remainder is 1 , Other individuals copy the emperor's first component with probability Pc, self mutation with probability Pm. 50 individuals get their respective SVM classification accuracy, choose the highest classification accuracy of the individual as emperor, gen $=$ gen +1 , if gen $/ 3$ of the remainder is 2 , other individuals copy the emperor's second component with probability Pc, Self mutation with probability Pm. 50 individuals get their respective SVM classification accuracy, choose the highest classification accuracy of the individual as emperor, gen $=$ gen +1 , if gen/3 of the remainder is 0 , other individuals copy the emperor's third component with probability Pc, Self mutation with probability Pm. until the number of cycles is reached. Record feature subset, window length and optimization algorithm of emperor. The classification prediction accuracy of emperor is the correct classification prediction accuracy.

Cooperative coevolution algorithm and the code used by SVM:

gen $=0$

for each species $\mathrm{s}$ do begin

Pop $_{\text {s }}$ (gen) $=$ randomly initialized each species' three subcomponents

end

while gen $<$ maximum number of generations do begin

$$
\text { gen }=\text { gen }+1
$$

calculate accuracy of each species by CC-SVM in Pop $_{s}$ (gen)

emperor $=$ the species with maximum accuracy

for each species s do begin

remainder $=\mathrm{s} \% 3$

the remainder 's subcomponent of each species in a Pc probability= the remainder 's subcomponent of emperor

self variation of the remainder 's subcomponent of each species in a Pm probability

end

end

\section{Experiments}

\subsection{Experimental Design}

Feature candlestick de-noising: in order to better verify our proposed method in financial market, extract data from 3612 different stock data. These financial data are taken from Wind and other public channels. In order to establish our trading model, the obtained financial data needs to be de-noised. We use the method of selecting characterized candlesticks from the data to process the financial time series. Take the candlesticks of all the same forms in the time series and their associated data. For example, select all the Inverted hammer in the downward trend. $\mathrm{n}$ days data as dataset. Select $\mathrm{m}$ from the following indicators: DIF DEA MACD DMA AMA PSY CLOSE D RSI HIGH OPEN MA5 MA10 VOLUME LOW K UPPERLINE MIDDLELINE LOWLINE CCI J. Both $\mathrm{m}$ and $\mathrm{n}$ need to be selected in the process of SVM training test. Compared with the data that is not de-noised, the data volume with the characterized candlestick de-noising is smaller, and the classification accuracy is higher. When comparing the experiment, the data of the same size as the above experiment was selected randomly.

The dataset will be divided into two parts, the training set and the test set. The first 200 sets of data sets are used as training sets and the latter 1000 sets as test sets. The superiority of the characterized candlestick de-noising is verified by experiment.

During SVM training, this paper mainly includes three parts, feature subset, window length, optimization 
algorithm. The usual approach is to look for the best parts one by one. This approach does not take into account the relationship between components, and the best of each component is not globally optimal. The cooperative coevolution algorithm will optimize the above three parts. The experimental results show that the cooperative coevolution is applied to SVM, and the classification accuracy is higher. Let's say that the number of cycles is $\mathrm{C}, \mathrm{N}$ individuals constitute a population, feature subset, window length, optimization algorithm to be determined, day is the number of days, fea represents a subset of features, and opt represents the choice of optimization algorithm. Initialize the first $\mathrm{k}$ individuals, the extraction of day is a random value between 5-30, fea is randomly take out the characteristics of 21 feature subset, which is take out the several characteristics and here several characteristics are random. Opt is a random number between 1-3, representing grid algorithm, genetic algorithm, particle swarm algorithm. Based on the three components of each individual in the population, the SVM based on the candle chart combines the characterized candlestick to noise, and CCEA and SVM can work together to get the accuracy.

Three components and accuracy are obtained as follows:

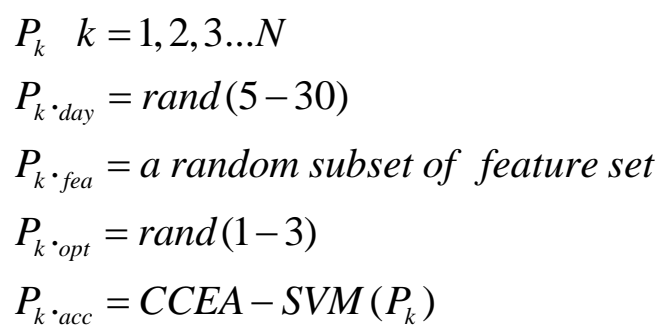

After the initialization is completed, each individual's feature subset, window length, optimization algorithm and accuracy will be obtained. The first three components of the cooperative coevolution algorithm are part 1 , part 2 , and part 3 . Accuracy as fitness. $\mathrm{N}$ individuals make up a population. Remove the highest accuracy of individuals for the emperor. If the current number of cycles $\mathrm{C} / 3$ remainder is 1 , except emperor, component 1 of the rest of the individual will be copied into component 1 of emperor according to the crossover rate $\mathrm{Pc}$, and then the variation of component 1 will be carried out according to the mutation rate $\mathrm{Pm}$. Then calculate the accuracy of each individual, take out the highest accuracy of the individual is emperor, if the current number of cycles $\mathrm{C} / 3$ remainder is 2 , except emperor, the rest of the individual parts 2 will be copied according to the crossover rate of Pc into emperor parts 2, after the completion of the copy, then according to the variation rate of Pm for their own variation. Then calculate the accuracy of each individual, the highest accuracy of the individual is emperor, if the current number of cycles $\mathrm{C} / 3$ remainder is 0 , then the other individual parts 3 will be copied into emperor parts 3 according to the crossover rate Pc, and then according to the mutation rate Pm to make their own variation. Reaching the maximum number of cycles, the last emperor is the individual we want.

\subsection{Experimental Results}

1) Comparison test between SVM and CANDEL+SVM to obtain the highest classification prediction accuracy step by step.

In the way of CANDEL+SVM, the first 200 sets of data are used as training sets, and the latter 1000 sets of data are used as test sets. The way of SVM is to randomly select the amount of data roughly equivalent to CANDEL+SVM from stock data.

Feature selection: select a better subset from 21 feature sets, and then predict classification accuracy of the first 1-21 feature subsets. In the following experiment, the characterized candlestick used in CANDEL+SVM is Inverted hammer. As shown in Fig. 3, CANDEL+SVM achieves higher classification accuracy, and the highest accuracy is the first seven times of the feature subset, which is $53.8740 \%$. SVM, with the 
first 14 feature sets of feature subset, obtained the highest classification accuracy of $52.5090 \%$. The de-noising of feature candlestick is helpful for obtaining higher classification accuracy. The feature set includes the DIF DEA MACD DMA AMA CLOSE D RSI HIGH OPEN MA5 MA10 VOLUME K LOW UPPERLINE MIDDLELINE LOWLINE CCI J, in order to rank the above features 1-21. If DIF DEA MACD DMA is taken out, it is expressed as 1.2.3. 4 in the table. The feature subset of the two methods is shown in Table 1. At this point, the number of days window is 1 , and the optimization algorithm selects the grid search algorithm in LIBSVM.

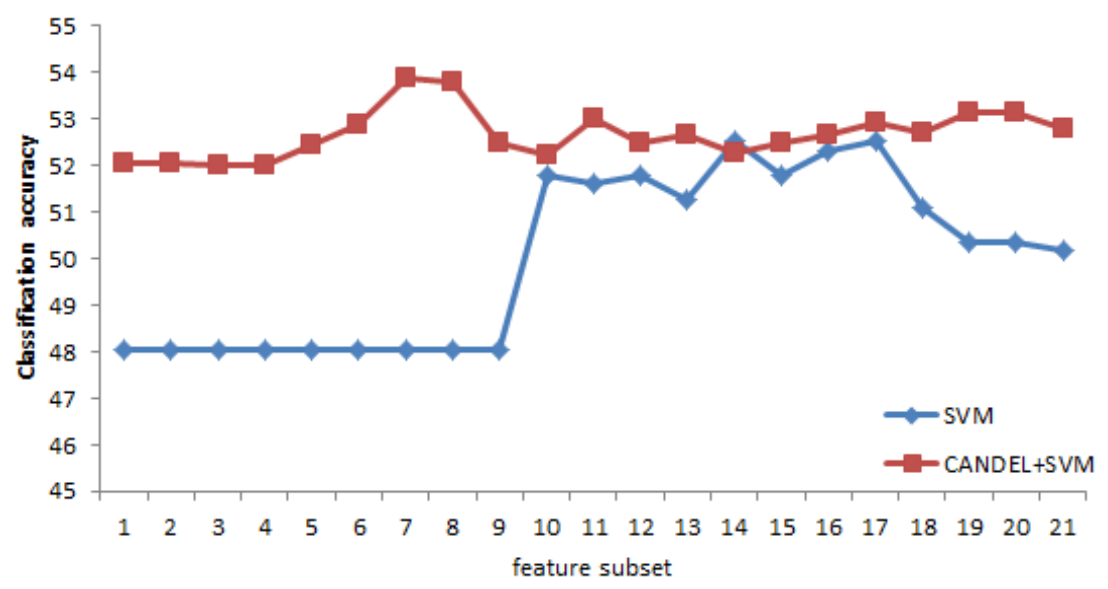

Fig. 3. Variation of classification accuracy with subsets.

Table 1. The Corresponding Feature Subset of the Optimal Classification Accuracy

\begin{tabular}{ccc}
\hline & feature subset & Accuracy\% \\
\hline SVM & 12.3 .4 .5 .6 .7 .8 .9 .10 .11 .12 .13 .14 & 52.5090 \\
SVM+CANDEL & 1.2 .3 .4 .5 .6 .7 & 53.8740 \\
\hline
\end{tabular}

Number of days window setting: As shown in Fig. 4, SVM+CANDEL obtained the maximum classification accuracy of $55.1537 \%$ at $n=16$. SVM, the maximum classification accuracy at $n=12$ was $53.7634 \%$. At this time, the feature subset is in Table 1, and the optimization algorithm is the grid search by LIBSVM.

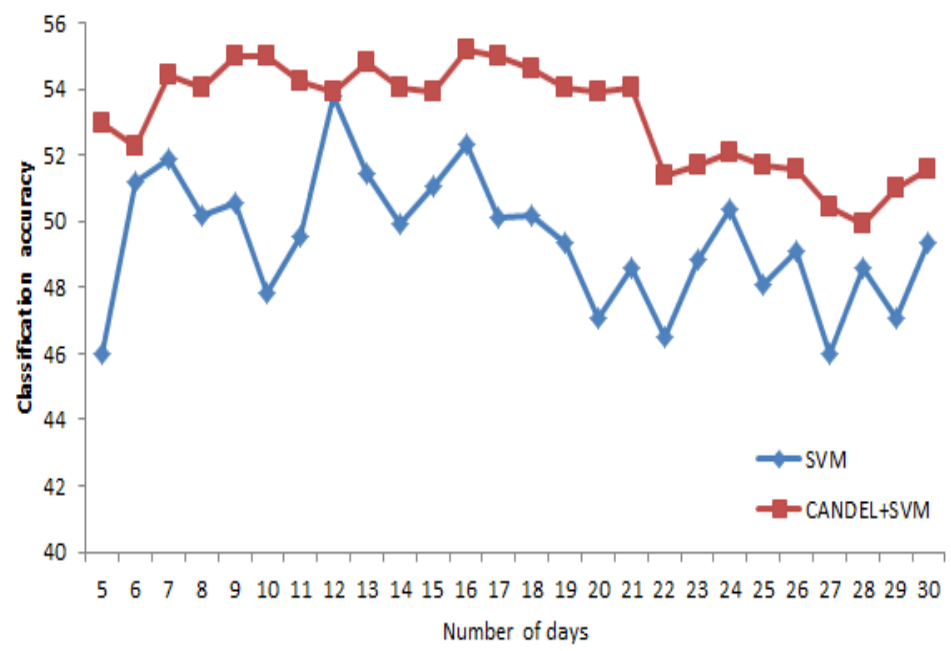

Fig. 4. Variation of the classification prediction accuracy with the number of days.

Optimization algorithm selection: As shown in Fig. 5 below, SVM+CANDEL achieves the highest predictive classification accuracy of 55. $6842 \%$ when selecting GA. SVM obtained the highest predictive classification 
accuracy $=53.7634 \%$ when $n=1$ and select grid search. At this time, the feature subset is in Table 1 , the number of days window of SVM is taken from the 12 obtained from 2. 2, and the window size of SVM+CANDEL is taken from the 16 obtained from 2. 2.

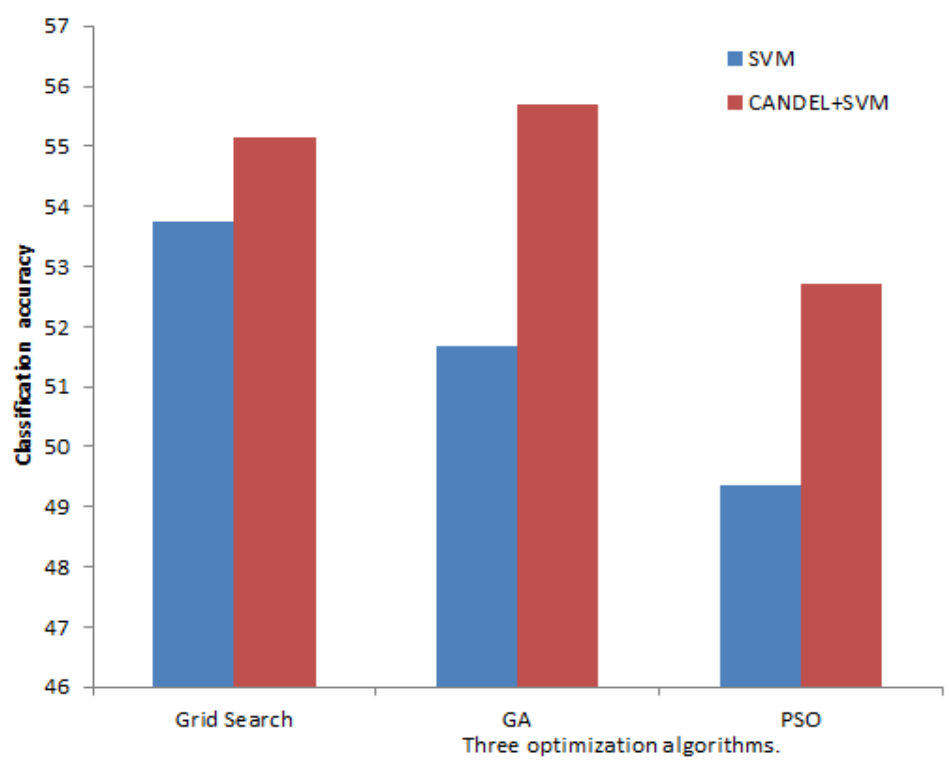

Fig. 5. The influence of three optimization algorithms on accuracy.

Table 2. SVM and CANDEL+SVM in 30 Different Feature Candlesticks, Three Optimization Algorithms

Predict Classification Accuracy

\begin{tabular}{|c|c|c|c|c|c|c|c|}
\hline \multirow[b]{2}{*}{ Pattern } & \multicolumn{4}{|l|}{ SVM } & \multicolumn{3}{|l|}{ CANDEL+SVM } \\
\hline & Grid Search & & $\mathrm{GA}$ & PSO & Grid Search & $\mathrm{GA}$ & PSO \\
\hline Inverted hammer & 53. 7634 & 51. & 6876 & 49. 3542 & 55. 1537 & 55. 6842 & 52. 7253 \\
\hline Hammer & 50. 9686 & 51. & 1830 & 49. 2805 & 49. 0606 & 49. 2470 & 47. 3252 \\
\hline Belt-hold line & 49. 7557 & 46. & 3225 & 46. 1177 & 52. 9579 & 52. 9579 & 52. 9579 \\
\hline Engulfing Patterns & 51. 9208 & 46. & 5430 & 53. 4789 & 50. 2800 & 51. 1516 & 50. 2905 \\
\hline Harami & 51. 4343 & 49. & 9645 & 47. 2705 & 57. 4875 & 57. 4875 & 57. 4875 \\
\hline Piercing pattern & 52. 8683 & 46. & 7351 & 46. 8072 & 54. 8983 & 52. 6802 & 52. 7418 \\
\hline Doji star & 52. 7710 & 52. & 2179 & 48. 8303 & 54. 0138 & 53. 7480 & 53. 6948 \\
\hline Pigeons form & 48. 5396 & 52. & 2134 & 47. 5057 & 52. 0328 & 52. 0328 & 52. 0328 \\
\hline White a soldier & 51. 3105 & 51. & 4905 & 49. 7216 & 53. 3930 & 52. 2744 & 50. 0373 \\
\hline Within the three rise & 47. 5034 & 47. & 1390 & 48. 5801 & 53. 6595 & 53. 6595 & 53. 6595 \\
\hline Without the three rise & 46. 2321 & 51. & 0130 & 53. 2324 & 48. 5072 & 48. 5072 & 48. 5072 \\
\hline Squeeze the alarm & 51. 6550 & 49. & 9413 & 52. 9945 & 57. 9794 & 57. 9794 & 57. 9794 \\
\hline Three gaps & 49. 8002 & 53. & 3946 & 46. 4003 & 63. 8258 & 63. 0682 & 63. 4470 \\
\hline Jump up and tie the Yin and Yang line. & 49. 6474 & 50. & 9323 & 51. 6077 & 62. 5000 & 58. 2746 & 57. 3944 \\
\hline Postwar dressing & 52. 87519 & 52. & 0825 & 48. 0453 & 57. 4335 & 57. 4335 & 57. 4335 \\
\hline Hanging man & 50. 6350 & 49. & 4489 & 49. 2136 & 55. 0206 & 55. 0206 & 55. 0206 \\
\hline belt-hold lines & 50. 6943 & 49. & 2862 & 50. 1638 & 52. 2047 & 51. 6672 & 51. 9069 \\
\hline Engulfing Pattern & 52. 5318 & 52. & 2724 & 53. 1648 & 51. 6262 & 55. 0000 & 55. 0087 \\
\hline Harami & 52. 1217 & 46. & 6344 & 49. 1749 & 59. 2556 & 57. 8518 & 59. 3862 \\
\hline Harami cros & 50. 3831 & 47. & 0121 & 53. 4712 & 62. 2523 & 62. 7928 & 63. 6036 \\
\hline Meteor line & 47. 3902 & 47. & 3178 & 48. 2911 & 49. 6134 & 50. 5300 & 51. 6180 \\
\hline Dark-cloud cover & 47. 8235 & 48. & 9711 & 51. 3284 & 54. 2543 & 54. 3043 & 52. 8028 \\
\hline Doji star & 52. 7375 & 52. & 3185 & 51. 0642 & 49. 1613 & 49. 1613 & 49. 2903 \\
\hline Swooping eagle & 46. 2179 & 52. & 1056 & 50. 0974 & 57. 0737 & 57. 0737 & 57. 0737 \\
\hline A black crow & 49. 7233 & 46. & 4596 & 51. 3056 & 59. 2556 & 57. 8518 & 59. 3862 \\
\hline Within the three drop & 47. 2762 & 49. & 0344 & 51. 0656 & 57. 9133 & 57. 9133 & 57. 9133 \\
\hline Without the three drop & 53. 4380 & 50. & 0043 & 47. 3538 & 54. 1641 & 51. 5731 & 52. 7452 \\
\hline Squeeze the alarm & 51. 4165 & 49. & 1677 & 46. 9729 & 54. 2842 & 54. 2842 & 54. 2842 \\
\hline Insert & 49. 8036 & 50. & 9921 & 53. 5930 & 52. 4354 & 52. 4354 & 52. 4354 \\
\hline Jump down and tie the Yin and Yang line. & 49. 5803 & 50. & 7726 & 47. 3005 & 52. 8736 & 52. 6820 & 51. 1494 \\
\hline
\end{tabular}

Table 2 is the prediction classification accuracy of the three optimization algorithms of SVM and CANDEL+SVM in different characterized candlesticks in 30. The first 15 are bullish in the downward trend, 
and the latter 15 are bearish patterns in the rising trend (bold, diagonal).

2) The comparison of CANDEL+SVM and CANDEL+CC-SVM in 30 characterized candlesticks.

The following Table 3 is the selected feature subset, number of days window and optimization algorithm in the prediction of 30 characterized candlesticks. The first 15 are bullish in the downward trend, and the latter 15 are bearish patterns in the rising trend (bold, diagonal). The feature set includes the DIF DEA MACD DMA AMA CLOSE D RSI HIGH OPEN MA5 MA10 VOLUME K LOW UPPERLINE MIDDLELINE LOWLINE CCI J, in order to rank the above features 1-21. If DIF DEA MACD DMA is taken out, it is expressed as 1.2. 3. 4 or 1-4 in the table.

Table 3. 30 Different Morphologies, Respectively in SVM+CANDEL and CC-SVM +CANDEL, Finally Acquired the Feature Subset, Number of Days Window, Optimization Algorithm and Accuracy

\begin{tabular}{|c|c|c|c|c|c|}
\hline Pattern & SVM type & characteristic subset & $\begin{array}{l}\text { Number of days } \\
\text { window }\end{array}$ & $\begin{array}{l}\text { optimization } \\
\text { algorithm }\end{array}$ & accuracy rate \\
\hline \multirow[t]{2}{*}{ Inverted hammer } & SVM+CANDEL & 1 & 18 & 1 & 55.6842 \\
\hline & CC-SVM+CANDEL & 16 & 28 & 2 & 55. 9364 \\
\hline \multirow[t]{2}{*}{ Hammer } & SVM+CANDEL & $1-20$ & 28 & 2 & 49. 2470 \\
\hline & CC-SVM+CANDEL & 1111519 & 28 & 1 & 55.7000 \\
\hline \multirow[t]{2}{*}{ Belt-hold line } & SVM+CANDEL & $1-6$ & 2 & 1 & 52. 9579 \\
\hline & CC-SVM+CANDEL & 416 & 30 & 2 & 53.0909 \\
\hline \multirow[t]{2}{*}{ Engulfing Patterns } & SVM+CANDEL & $1-17$ & 25 & 2 & 51. 1516 \\
\hline & CC-SVM+CANDEL & 1715 & 29 & 2 & 57.2222 \\
\hline \multirow[t]{2}{*}{ Harami } & SVM+CANDEL & 1 & 1 & 1 & 57.4875 \\
\hline & CC-SVM+CANDEL & 1715 & 26 & 1 & 56.6250 \\
\hline \multirow[t]{2}{*}{ Piercing pattern } & SVM+CANDEL & $1-13$ & 29 & 1 & 54. 8983 \\
\hline & CC-SVM+CANDEL & 61020 & 29 & 1 & 56.8000 \\
\hline \multirow[t]{2}{*}{ Doji star } & SVM+CANDEL & $1-20$ & 19 & 1 & 54.0138 \\
\hline & CC-SVM+CANDEL & 131517 & 26 & 1 & 56.7000 \\
\hline \multirow[t]{2}{*}{ Pigeons form } & SVM+CANDEL & $1-7$ & 1 & 1 & 52.0328 \\
\hline & CC-SVM+CANDEL & 12371112141520 & 27 & 2 & 58.4000 \\
\hline \multirow[t]{2}{*}{ White a soldier } & SVM+CANDEL & $1-21$ & 16 & 1 & 53.3930 \\
\hline & CC-SVM+CANDEL & $1,7,8,10,11,14,15,18,19,20$ & 27 & 2 & 58.3000 \\
\hline \multirow[t]{2}{*}{ Within the three rise } & SVM+CANDEL & $1-7$ & 1 & 1 & 53.6595 \\
\hline & CC-SVM+CANDEL & $5,7,16,18,19,20$ & 29 & 1 & 57. 3000 \\
\hline \multirow[t]{2}{*}{ Without the three rise } & SVM+CANDEL & $1-5$ & 1 & 1 & 48. 5072 \\
\hline & CC-SVM+CANDEL & 1,4 & 26 & 1 & 54.7000 \\
\hline \multirow[t]{2}{*}{ Squeeze the alarm } & SVM+CANDEL & 1 & 1 & 1 & 57. 9794 \\
\hline & CC-SVM+CANDEL & 2,11 & 30 & 2 & 58.7273 \\
\hline \multirow[t]{2}{*}{ Three gaps } & SVM+CANDEL & 1 & 22 & 1 & 63.8258 \\
\hline & CC-SVM+CANDEL & $1-9,11-21$ & 26 & 3 & 64.6250 \\
\hline \multirow[t]{2}{*}{ Jump up and tie the Yin and Yang line. } & SVM+CANDEL & $1-10$ & 23 & 1 & 62.5000 \\
\hline & CC-SVM+CANDEL & $4,8,16,20$ & 26 & 1 & 66.1250 \\
\hline \multirow[t]{2}{*}{ Postwar dressing } & SVM+CANDEL & $1-6$ & 1 & 1 & 57.4335 \\
\hline & CC-SVM+CANDEL & $11,13,14,20$ & 29 & 3 & 58.7000 \\
\hline \multirow[t]{2}{*}{ Hanging man } & SVM+CANDEL & $1-9$ & 1 & 1 & 55.0206 \\
\hline & CC-SVM+CANDEL & 5,15 & 26 & 1 & 57.7000 \\
\hline belt-hold lines & SVM+CANDEL & $1-19$ & 4 & 1 & 52. 2047 \\
\hline & CC-SVM+CANDEL & $1,4,6,8,15,17,18,21$ & 28 & 1 & 53.8000 \\
\hline Engulfing Pattern & SVM+CANDEL & 123 & 17 & 3 & 55.0087 \\
\hline & CC-SVM+CANDEL & $1-10,121314162021$ & 30 & 1 & 57.5000 \\
\hline Harami & SVM+CANDEL & $1-7$ & 30 & 3 & 59. 3862 \\
\hline & CC-SVM+CANDEL & $1,2,4,6,14$ & 30 & 2 & 55.5000 \\
\hline Harami cros & SVM+CANDEL & $1-21$ & 29 & 3 & 63.6036 \\
\hline & CC-SVM+CANDEL & $1-5,8,11-15,18192021$ & 26 & 1 & 63.3000 \\
\hline Meteor line & SVM+CANDEL & $1-6$ & 29 & 3 & 51.6180 \\
\hline & CC-SVM+CANDEL & $5,7,9,11,12,13$ & 28 & 1 & 56.2000 \\
\hline Dark-cloud cover & SVM+CANDEL & $1-21$ & 19 & 2 & 54. 3043 \\
\hline & CC-SVM+CANDEL & $2-8,10,11-18,20$ & 26 & 1 & 54. 5000 \\
\hline Doji star & SVM+CANDEL & $1-21$ & 26 & 3 & 49. 2903 \\
\hline & CC-SVM+CANDEL & 1,11 & 30 & 2 & 56.2000 \\
\hline Swooping eagle & SVM+CANDEL & $1-5$ & 1 & 1 & 57.0737 \\
\hline & CC-SVM+CANDEL & 3791516 & 30 & 1 & 58.3886 \\
\hline A black crow & SVM+CANDEL & $1-7$ & 30 & 3 & 59. 3862 \\
\hline & CC-SVM+CANDEL & 13,17 & 29 & 1 & 61.8000 \\
\hline Within the three drop & SVM+CANDEL & $1-9$ & 1 & 1 & 57.9133 \\
\hline & CC-SVM+CANDEL & 3 & 26 & 1 & 60.6000 \\
\hline Without the three drop & SVM+CANDEL & $1-7$ & 19 & 1 & 54. 1641 \\
\hline & CC-SVM+CANDEL & $1-18,2021$ & 30 & 2 & 59.4000 \\
\hline Squeeze the alarm & SVM+CANDEL & $1-7$ & 1 & 1 & 54. 2842 \\
\hline & CC-SVM+CANDEL & $3,5,9,11,20$ & 28 & 1 & 55.0000 \\
\hline Insert & SVM+CANDEL & $1-6$ & 1 & 1 & 52.4354 \\
\hline & CC-SVM+CANDEL & $5,10,12,14,15,18,19$ & 26 & 1 & 59. 3000 \\
\hline Jump down and tie the Yin and Yang line. & SVM+CANDEL & & 3 & 1 & 52.8736 \\
\hline & CC-SVM+CANDEL & $2,5,6,11,13,14,15$ & 29 & 2 & 57.2222 \\
\hline
\end{tabular}

In CANDEL+ CC-SVM, with the increase of genetic algebra, the prediction accuracy rate of the 30 characterized candlesticks was changed as follows. Bullish in the downward trend lines to Inverted hammer, Hammer, Belt-hold line, Engulfing Patterns, Harami, Piercing pattern, Doji star, Pigeons form, White a soldier, Within the three rise, Without the three rise, Squeeze the alarm, Three gaps, Jump up and tie the Yin and Yang line, Postwar dressing, respectively, with $1+2+, 3+\ldots 15+$ said. With the increase of the cyclic algebra G, the change of accuracy is shown in Table 4. Bearish in the rising trend of Hanging man, Belt-hold 
lines, Engulfing Pattern, Harami, Harami cros, Meteor line, Dark-cloud cover, Doji star, Swooping eagle, A black crow, Within the three drop, Without the three drop, Squeeze the alarm, Insert, Jump down and tie the Yin and Yang line, respectively, with 1 -, 2 -, 3 -. . 15 - said. With the increase of the cyclic algebra G, the change of accuracy is shown in Table 5.

Table 4. Shows the 15 Characterized Candlesticks of the Rising Trend, and the Accuracy Changes with the Increase of Iteration Number G

\begin{tabular}{|c|c|c|c|c|c|c|c|c|c|c|c|c|c|c|c|}
\hline G & $1+$ & $2+$ & $3+$ & $4+$ & $5+$ & $6+$ & $7+$ & $8+$ & $9+$ & $10+$ & $11+$ & $12+$ & $13+$ & $14+$ & $15+$ \\
\hline 1 & 53.3636 & 52.6000 & 52.1818 & 55.2222 & 55.7500 & 53.3000 & 53.4000 & 57.0000 & 57.9000 & 57.1000 & 53.5000 & 55.8182 & 64.1250 & 64.8750 & 56.6000 \\
\hline 2 & 53.3636 & 52.6000 & 52.1818 & 57.2222 & 56.3750 & 53.3000 & 53.6000 & 57.2000 & 57.9000 & 57.1000 & 53.6000 & 56.0000 & 64.3750 & 64.8750 & 56.6000 \\
\hline 3 & 53.3636 & 52.6000 & 52.1818 & 57.2222 & 56.3750 & 53.9000 & 53.7000 & 57.2000 & 57.9000 & 57.1000 & 53.6000 & 56.1818 & 64.3750 & 65.8750 & 56.6000 \\
\hline 4 & 53.8182 & 52.6000 & 52.1818 & 57.2222 & 56.3750 & 53.9000 & 53.7000 & 57.8000 & 57.9000 & 57.3000 & 53.6000 & 56.1818 & 64.3750 & 65.8750 & 56.6000 \\
\hline 5 & 53.9091 & 52.6000 & 52.1818 & 57.2222 & 56.3750 & 54.3000 & 55.8000 & 57.8000 & 57.9000 & 57.3000 & 53.6000 & 57.6364 & 64.3750 & 65.8750 & 56.6000 \\
\hline 6 & 55.6364 & 52.6000 & 52.6364 & 57.2222 & 56.3750 & 54.3000 & 55.8000 & 57.8000 & 57.9000 & 57.3000 & 53.6000 & 57.6364 & 64.3750 & 65.8750 & 56.6000 \\
\hline 7 & 55.9364 & 52.6000 & 52.6364 & 57.2222 & 56.6250 & 54.4000 & 55.8000 & 58.1000 & 57.9000 & 57.3000 & 53.6000 & 57.6364 & 64.3750 & 65.8750 & 56.6000 \\
\hline 8 & 55.9364 & 52.6000 & 52.6364 & 57.2222 & 56.6250 & 54.9000 & 55.8000 & 58.1000 & 57.9000 & 57.3000 & 53.6000 & 58.0000 & 64.5000 & 65.8750 & 56.6000 \\
\hline 9 & 55.9364 & 52.6000 & 52.8182 & 57.2222 & 56.6250 & 54.9000 & 55.8000 & 58.1000 & 58.3000 & 57.3000 & 53.6000 & 58.0000 & 64.5000 & 65.8750 & 56.6000 \\
\hline 10 & 55.9364 & 52.6000 & 52.8182 & 57.2222 & 56.6250 & 55.1000 & 56.1000 & 58.1000 & 58.3000 & 57.3000 & 53.6000 & 58.7273 & 64.5000 & 65.8750 & 56.6000 \\
\hline 11 & 55.9364 & 52.6000 & 52.8182 & 57.2222 & 56.6250 & 55.6000 & 56.1000 & 58.1000 & 58.3000 & 57.3000 & 53.6000 & 58.7273 & 64.5000 & 65.8750 & 57.8000 \\
\hline 12 & 55.9364 & 52.6000 & 52.9091 & 57.2222 & 56.6250 & 55.6000 & 56.1000 & 58.1000 & 58.3000 & 57.3000 & 53.6000 & 58.7273 & 64.5000 & 65.8750 & 57.8000 \\
\hline 13 & 55.9364 & 52.6000 & 52.9091 & 57.2222 & 56.6250 & 56.8000 & 56.1000 & 58.1000 & 58.3000 & 57.3000 & 53.6000 & 58.7273 & 64.5000 & 65.8750 & 57.8000 \\
\hline 14 & 55.9364 & 52.6000 & 52.9091 & 57.2222 & 56.6250 & 56.8000 & 56.1000 & 58.1000 & 58.3000 & 57.3000 & 53.9000 & 58.7273 & 64.6250 & 65.8750 & 57.8000 \\
\hline 15 & 55.9364 & 52.6000 & 52.9091 & 57.2222 & 56.6250 & 56.8000 & 56.1000 & 58.1000 & 58.3000 & 57.3000 & 53.9000 & 58.7273 & 64.6250 & 65.8750 & 57.8000 \\
\hline 16 & 55.9364 & 52.6000 & 52.9091 & 57.2222 & 56.6250 & 56.8000 & 56.1000 & 58.1000 & 58.3000 & 57.3000 & 53.9000 & 58.7273 & 64.6250 & 65.8750 & 57.8000 \\
\hline 17 & 55.9364 & 54.8000 & 53.0000 & 57.2222 & 56.6250 & 56.8000 & 56.1000 & 58.1000 & 58.3000 & 57.3000 & 53.9000 & 58.7273 & 64.6250 & 65.8750 & 57.8000 \\
\hline 18 & 55.9364 & 54.8000 & 53.0000 & 57.2222 & 56.6250 & 56.8000 & 56.1000 & 58.1000 & 58.3000 & 57.3000 & 53.9000 & 58.7273 & 64.6250 & 65.8750 & 57.8000 \\
\hline 19 & 55.9364 & 54.8000 & 53.0000 & 57.2222 & 56.6250 & 56.8000 & 56.1000 & 58.1000 & 58.3000 & 57.3000 & 53.9000 & 58.7273 & 64.6250 & 65.8750 & 57.8000 \\
\hline 20 & 55.9364 & 54.8000 & 53.0000 & 57.2222 & 56.6250 & 56.8000 & 56.1000 & 58.1000 & 58.3000 & 57.3000 & 54.6000 & 58.7273 & 64.6250 & 65.8750 & 57.8000 \\
\hline 21 & 55.9364 & 54.8000 & 53.0000 & 57.2222 & 56.6250 & 56.8000 & 56.1000 & 58.1000 & 58.3000 & 57.3000 & 54.6000 & 58.7273 & 64.6250 & 65.8750 & 58.2000 \\
\hline 22 & 55.9364 & 55.7000 & 53.0000 & 57.2222 & 56.6250 & 56.8000 & 56.1000 & 58.1000 & 58.3000 & 57.3000 & 54.6000 & 58.7273 & 64.6250 & 65.8750 & 58.2000 \\
\hline 23 & 55.9364 & 55.7000 & 53.0000 & 57.2222 & 56.6250 & 56.8000 & 56.1000 & 58.1000 & 58.3000 & 57.3000 & 54.6000 & 58.7273 & 64.6250 & 65.8750 & 58.2000 \\
\hline 24 & 55.9364 & 55.7000 & 53.0000 & 57.2222 & 56.6250 & 56.8000 & 56.1000 & 58.1000 & 58.3000 & 57.3000 & 54.6000 & 58.7273 & 64.6250 & 65.8750 & 58.2000 \\
\hline 25 & 55.9364 & 55.7000 & 53.0000 & 57.2222 & 56.6250 & 56.8000 & 56.1000 & 58.1000 & 58.3000 & 57.3000 & 54.7000 & 58.7273 & 64.6250 & 66.1250 & 58.2000 \\
\hline 26 & 55.9364 & 55.7000 & 53.0000 & 57.2222 & 56.6250 & 56.8000 & 56.1000 & 58.1000 & 58.3000 & 57.3000 & 54.7000 & 58.7273 & 64.6250 & 66.1250 & 58.2000 \\
\hline 27 & 55.9364 & 55.7000 & 53.0000 & 57.2222 & 56.6250 & 56.8000 & 56.1000 & 58.1000 & 58.3000 & 57.3000 & 54.7000 & 58.7273 & 64.6250 & 66.1250 & 58.7000 \\
\hline 28 & 55.9364 & 55.7000 & 53.0000 & 57.2222 & 56.6250 & 56.8000 & 56.1000 & 58.1000 & 58.3000 & 57.3000 & 54.7000 & 58.7273 & 64.6250 & 66.1250 & 58.7000 \\
\hline 29 & 55.9364 & 55.7000 & 53.0000 & 57.2222 & 56.6250 & 56.8000 & 56.7000 & 58.4000 & 58.3000 & 57.3000 & 54.7000 & 58.7273 & 64.6250 & 66.1250 & 58.7000 \\
\hline 30 & 55.9364 & 55.7000 & 53.0909 & 57.2222 & 56.6250 & 56.8000 & 56.7000 & 58.4000 & 58.3000 & 57.3000 & 54.7000 & 58.7273 & 64.6250 & 66.1250 & 58.7000 \\
\hline
\end{tabular}

Table 5. The 15 Characterized Candlesticks of the Downward Trend, the Accuracy Changes with the Increment of the Iteration Number G

\begin{tabular}{|c|c|c|c|c|c|c|c|c|c|c|c|c|c|c|c|}
\hline \multicolumn{16}{|l|}{$\mathrm{G}$} \\
\hline & 1- & $2-$ & 3- & 4- & 5- & $6-$ & $7-$ & 8- & 9- & 10- & 11- & $12-$ & 13- & $14-$ & $15-$ \\
\hline 1 & 56.9000 & 51.7000 & 56.6000 & 53.7000 & 62.9000 & 53.8000 & 54.0000 & 52.9000 & 54.2859 & 59.9000 & 60.1000 & 59.2000 & 54.4000 & 58.4000 & 55.2222 \\
\hline 2 & 56.9000 & 52.7000 & 57.1000 & 53.7000 & 62.9000 & 54.4000 & 54.0000 & 52.9000 & 54.3205 & 59.9000 & 60.1000 & 59.2000 & 54.4000 & 58.6000 & 55.2222 \\
\hline 3 & 57.1000 & 52.7000 & 57.1000 & 53.7000 & 62.9000 & 54.4000 & 54.0000 & 55.1000 & 56.3740 & 59.9000 & 60.1000 & 59.2000 & 55.0000 & 58.6000 & 55.4444 \\
\hline 4 & 57.1000 & 52.9000 & 57.1000 & 53.7000 & 62.9000 & 54.4000 & 54.0000 & 55.1000 & 56.8056 & 60.1000 & 60.1000 & 59.2000 & 55.0000 & 58.6000 & 55.6667 \\
\hline
\end{tabular}




\begin{tabular}{|c|c|c|c|c|c|c|c|c|c|c|c|c|c|c|c|}
\hline 5 & 57.1000 & 52.9000 & 57.4000 & 54.2000 & 62.9000 & 54.5000 & 54.3000 & 55.9000 & 56.9430 & 61.8000 & 60.3000 & 59.2000 & 55.0000 & 58.6000 & 56.0000 \\
\hline 6 & 57.1000 & 52.9000 & 57.4000 & 54.2000 & 62.9000 & 54.6000 & 54.3000 & 55.9000 & 57.2689 & 61.8000 & 60.6000 & 59.3000 & 55.0000 & 58.6000 & 56.0000 \\
\hline 7 & 57.1000 & 52.9000 & 57.5000 & 54.2000 & 62.9000 & 54.6000 & 54.3000 & 55.9000 & 57.2689 & 61.8000 & 60.6000 & 59.3000 & 55.0000 & 58.6000 & 56.1111 \\
\hline 8 & 57.1000 & 53.8000 & 57.5000 & 54.2000 & 62.9000 & 54.6000 & 54.3000 & 56.0000 & 58.3651 & 61.8000 & 60.6000 & 59.4000 & 55.0000 & 58.6000 & 56.1111 \\
\hline 9 & 57.1000 & 53.8000 & 57.5000 & 54.2000 & 62.9000 & 54.7000 & 54.3000 & 56.0000 & 58.3651 & 61.8000 & 60.6000 & 59.4000 & 55.0000 & 58.6000 & 56.1111 \\
\hline 10 & 57.1000 & 53.8000 & 57.5000 & 54.6000 & 62.9000 & 54.7000 & 54.3000 & 56.0000 & 58.3651 & 61.8000 & 60.6000 & 59.4000 & 55.0000 & 58.6000 & 56.1111 \\
\hline 11 & 57.5000 & 53.8000 & 57.5000 & 54.8000 & 62.9000 & 54.8000 & 54.3000 & 56.0000 & 58.3651 & 61.8000 & 60.6000 & 59.4000 & 55.0000 & 59.3000 & 56.1111 \\
\hline 12 & 57.5000 & 53.8000 & 57.5000 & 54.8000 & 62.9000 & 54.8000 & 54.3000 & 56.0000 & 58.3651 & 61.8000 & 60.6000 & 59.4000 & 55.0000 & 59.3000 & 56.1111 \\
\hline 13 & 57.7000 & 53.8000 & 57.5000 & 54.8000 & 62.9000 & 54.8000 & 54.3000 & 56.0000 & 58.3886 & 61.8000 & 60.6000 & 59.4000 & 55.0000 & 59.3000 & 56.3333 \\
\hline 14 & 57.7000 & 53.8000 & 57.5000 & 54.8000 & 62.9000 & 55.3000 & 54.5000 & 56.0000 & 58.3886 & 61.8000 & 60.6000 & 59.4000 & 55.0000 & 59.3000 & 56.3333 \\
\hline 15 & 57.7000 & 53.8000 & 57.5000 & 54.8000 & 62.9000 & 55.3000 & 54.5000 & 56.0000 & 58.3886 & 61.8000 & 60.6000 & 59.4000 & 55.0000 & 59.3000 & 56.3333 \\
\hline 16 & 57.7000 & 53.8000 & 57.5000 & 54.8000 & 62.9000 & 55.3000 & 54.5000 & 56.0000 & 58.3886 & 61.8000 & 60.6000 & 59.4000 & 55.0000 & 59.3000 & 56.3333 \\
\hline 17 & 57.7000 & 53.8000 & 57.5000 & 54.8000 & 62.9000 & 55.3000 & 54.5000 & 56.0000 & 58.3886 & 61.8000 & 60.6000 & 59.4000 & 55.0000 & 59.3000 & 56.3333 \\
\hline 18 & 57.7000 & 53.8000 & 57.5000 & 55.5000 & 62.9000 & 55.3000 & 54.5000 & 56.0000 & 58.3886 & 61.8000 & 60.6000 & 59.4000 & 55.0000 & 59.3000 & 56.3333 \\
\hline 19 & 57.7000 & 53.8000 & 57.5000 & 55.5000 & 62.9000 & 55.3000 & 54.5000 & 56.0000 & 58.3886 & 61.8000 & 60.6000 & 59.4000 & 55.0000 & 59.3000 & 56.5556 \\
\hline 20 & 57.7000 & 53.8000 & 57.5000 & 55.5000 & 62.9000 & 56.2000 & 54.5000 & 56.0000 & 58.3886 & 61.8000 & 60.6000 & 59.4000 & 55.0000 & 59.3000 & 56.5556 \\
\hline 21 & 57.7000 & 53.8000 & 57.5000 & 55.5000 & 62.9000 & 56.2000 & 54.5000 & 56.0000 & 58.3886 & 61.8000 & 60.6000 & 59.4000 & 55.0000 & 59.3000 & 56.5556 \\
\hline 22 & 57.7000 & 53.8000 & 57.5000 & 55.5000 & 62.9000 & 56.2000 & 54.5000 & 56.0000 & 58.3886 & 61.8000 & 60.6000 & 59.4000 & 55.0000 & 59.3000 & 56.5556 \\
\hline 23 & 57.7000 & 53.8000 & 57.5000 & 55.5000 & 62.9000 & 56.2000 & 54.5000 & 56.0000 & 58.3886 & 61.8000 & 60.6000 & 59.4000 & 55.0000 & 59.3000 & 56.6667 \\
\hline 24 & 57.7000 & 53.8000 & 57.5000 & 55.5000 & 62.9000 & 56.2000 & 54.5000 & 56.2000 & 58.3886 & 61.8000 & 60.6000 & 59.4000 & 55.0000 & 59.3000 & 56.6667 \\
\hline 25 & 57.7000 & 53.8000 & 57.5000 & 55.5000 & 62.9000 & 56.2000 & 54.5000 & 56.2000 & 58.3886 & 61.8000 & 60.6000 & 59.4000 & 55.0000 & 59.3000 & 57.1111 \\
\hline 26 & 57.7000 & 53.8000 & 57.5000 & 55.5000 & 63.3000 & 56.2000 & 54.5000 & 56.2000 & 58.3886 & 61.8000 & 60.6000 & 59.4000 & 55.0000 & 59.3000 & 57.1111 \\
\hline 27 & 57.7000 & 53.8000 & 57.5000 & 55.5000 & 63.3000 & 56.2000 & 54.5000 & 56.2000 & 58.3886 & 61.8000 & 60.6000 & 59.4000 & 55.0000 & 59.3000 & 57.2222 \\
\hline 28 & 57.7000 & 53.8000 & 57.5000 & 55.5000 & 63.3000 & 56.2000 & 54.5000 & 56.2000 & 58.3886 & 61.8000 & 60.6000 & 59.4000 & 55.0000 & 59.3000 & 57.2222 \\
\hline 29 & 57.7000 & 53.8000 & 57.5000 & 55.5000 & 63.3000 & 56.2000 & 54.5000 & 56.2000 & 58.3886 & 61.8000 & 60.6000 & 59.4000 & 55.0000 & 59.3000 & 57.2222 \\
\hline 30 & 57.7000 & 53.8000 & 57.5000 & 55.5000 & 63.3000 & 56.2000 & 54.5000 & 56.2000 & 58.3886 & 61.8000 & 60.6000 & 59.4000 & 55.0000 & 59.3000 & 57.2222 \\
\hline
\end{tabular}

The classification accuracy of the three classification models of SVM CANDEL+SVM CANDEL+ CC-SVM in 30 characteristics of candlestick.

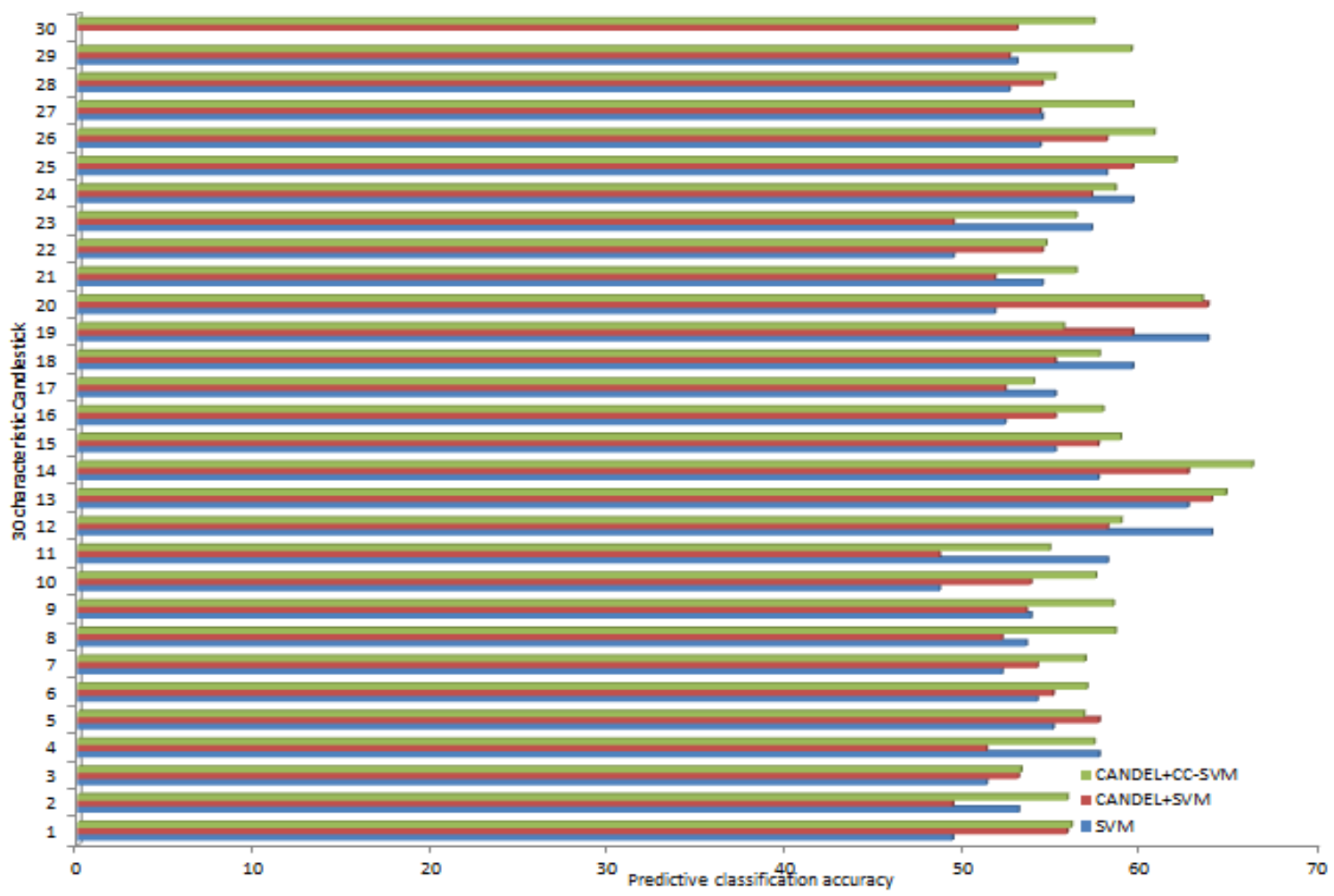

Fig. 6. Shows the classification accuracy of the three classification models of SVM CANDEL+SVM CANDEL+ CC-SVM in 30 characteristics of candlestick. 


\section{Conclusions}

This paper introduces a new forecasting model of financial time series, based on the classification prediction (SVM) model of feature candlestick and cooperative coevolution. characterized Candlestick financial time series reduces the data volume and improves the accuracy of prediction classification. Cooperative coevolution algorithm coordinates three parts in SVM training process, selection of feature subset, number of days window size and selection of optimization algorithm. The cooperative coevolution algorithm and SVM combine to fully consider the relationship between the three components, so that the three components coordinate and work together to achieve higher classification accuracy.

In the experiment, the data came from more than 3,000 stock data, covering the period from 1999 to 2016. Firstly, the data of financial time series with characteristic Candlestick is used to obtain all data that satisfy the particular characteristic Candlestick as the sample point to construct the SVM+CANDEL model. As a comparison, the traditional SVM prediction method was used to randomly select the same number of samples from the same 3,000 stocks as the CANDEL+SVM model. Through training and prediction process, the accuracy of classification prediction was obtained. The classification accuracy of the CANDEL+SVM model is generally higher than the classification prediction accuracy of the simple SVM model. The above comparison experiment verifies the validity of characteristic Candlestick financial time series on its trend prediction. Further, through the Candlestick financial time series, then the SVM combined with CCEA, coordinate the optimal feature subset selection, number of days window size set and optimization algorithm to choose the three parts, establish CANDEL + CC-SVM model, using the same training and testing data sets, to obtain the model prediction accuracy of classification, the characteristic of 30 different Candlestick, CANDEL + CC-SVM model of the classification accuracy is generally higher than the CANDEL + SVM classification prediction accuracy of the model. The above experiments prove that the prediction model of CANDEL+ CC-SVM can effectively improve the prediction accuracy of financial time series compared with traditional methods.

\section{Acknowledgement}

This research is supported by the National Natural Science Foundation of China (Nos: 61402267); Shandong Provincial Natural Science Foundation (ZR2014FQ004).

\section{Reference}

[1] He, J., Tan, A. H., \& Tan, C. L. (2003). On machine learning methods for Chinese document categorization. Applied Intelligence, 18(3), 311-322.

[2] Owusu, E., Zhan, Y., \& Mao, Q. R. (2014). An SVM-AdaBoost facial expression recognition system. Applied Intelligence, 40(3), 536-545.

[3] Hou, C., Nie, F., \& Zhang, C. (2014). Multiple rank multi-linear SVM for matrix data classification. Pattern Recognition, 47(1), 454-469.

[4] Castillo, C., Chollett, I., \& Klein, E. (2008). Enhanced duckweed detection using bootstrapped SVM classification on medium resolution RGB MODIS imagery. International Journal of Remote Sensing, 29(19), 5595-5604.

[5] Sirinukunwattana, K., Pluim, J. P., \& Chen, H. (2017). Gland segmentation in colon histology images: The glas challenge contest. Medical Image Analysis, 35, 489-502.

[6] Villarejo, L., Hernando, J., \& Padrell, J. (2006). Architecture and dialogue design for a voice operated information system. Applied Intelligence, 24(3), 253-261.

[7] Pons, E., Braun, L. M., \& Hunink, M. G. (2016). Natural language processing in radiology: A systematic review. Radiology, 279(2), 329. 
[8] Cambria, E., Xia, Y., \& White, B. New avenues in knowledge bases for natural language processing. Knowledge-Based Systems, 108(C), 1-4.

[9] Zhou, T., Gao, S., \& Wang, J. (2016). Financial time series prediction using a dendritic neuron model. Knowledge-Based Systems, 105(C), 214-224.

[10] Wang, L., \& Zhu, J. Financial market forecasting using a two-step kernel learning method for the support vector regression. Annals of Operations Research, 174(1), 103-120.

[11] He, H., \& Starzyk, J. A. (2014). A self-organizing learning array system for power quality classification based on wavelet transform. IEEE Transactions on Power Delivery, 21(1), 286-295.

[12] Lu, C., Lee, T., \& Chiu, C. (2009). Financial time series forecasting using independent component analysis and support vector regression. Decision Support Systems, 47(2), 115-125.

[13] Lee, K. H., \& Jo, G. S. (1999). Expert system for predicting stock market timing using a candlestick chart. Expert Systems with Applications, 16(4), 357-364.

[14] FISCHER. (1986). Noise. Journal of Finance, 41(3), 529-543.

[15] Kim, K. S., Zhao, Y., \& Jang, H. (2009). Large-scale pattern growth of graphene films for stretchable transparent electrodes. Nature, 457(7230), 706-710.

[16] Oliveira, F. B. D., Enayatifar, R., \& Sadaei, H. J. (2016). A cooperative coevolutionary algorithm for the multi-depot vehicle routing problem. Expert Systems with Applications An International Journal, 43(C), 117-130.

[17] Yang, Y., He, Z., \& Mabu, S. (2012). A cooperative coevolutionary stock trading model using genetic network programming-Sarsa. Journal of Advanced Computational Intelligence \& Intelligent Informatics, 16(5), 581-590.

[18] Rivera, A. J., García-Domingo, B., \& Jesus, M. J. D. (2013). Characterization of concentrating photovoltaic modules by cooperative competitive radial basis function networks. Expert Systems with Applications, 40(5), 1599-1608.

[19] Rabil, B, S., Tliba, S., \& Granger, E. (2013). Securing high resolution grayscale facial captures using a blockwise coevolutionary GA. Expert Systems with Applications An International Journal, 40(17), 6693-6706.

[20] Trunfio, G. A., Topa, P., \& Was, J. (2016). A new algorithm for adapting the configuration of subcomponents in large-scale optimization with cooperative coevolution. Information Sciences, 372, 773-795.

[21] Peng, X., Jin, Y., \& Wang, H. Multimodal optimization enhanced cooperative coevolution for large-scale optimization. IEEE Transactions on Cybernetics, 99.

[22] Sabar, N. R., Abawajy, J., \& Yearwood, J. Heterogeneous cooperative co-evolution memetic differential evolution algorithm for big data optimization problems. IEEE Transactions on Evolutionary Computation, 21(2), 315-327.

[23] Mahdavi, S., Rahnamayan, S., \& Shiri, M. E. Cooperative co-evolution with sensitivity analysis-based budget assignment strategy for large-scale global optimization. Applied Intelligence, 47(3), 888-913.

[24] Yang, M., Omidvar, M. N., \& Li, C. (2017). Supplementary file of efficient resource allocation in cooperative co-evolution for large-scale global optimization. IEEE Transactions on Evolutionary Computation, 99.

[25] Jia, Y. H., Chen, W. N., \& Gu, T. (2018). Distributed cooperative co-evolution with adaptive computing resource allocation for large scale optimization. IEEE Transactions on Evolutionary Computation, 99.

[26] Chandra, R., \& Zhang, M. (2015). Competition and collaboration in cooperative coevolution of elman recurrent neural networks for time-series prediction. IEEE Transactions on Neural Networks \& Learning Systems, 26(12), 3123. 
[27] Gibson, A. K., Stoy, K. S., \& Gelarden, I. A. (2016). The evolution of reduced antagonism-A role for hostparasite coevolution. Evolution, 69(11), 2820-2830.

[28] Mei, Y., Omidvar, M. N., \& Li, X. (2016). A competitive divide-and-conquer algorithm for unconstrained large-scale black-box optimization. Acm Transactions on Mathematical Software, 42(2), 13.

[29] Yoder, J. B. (2016). Understanding the coevolutionary dynamics of mutualism with population genomics. American Journal of Botany, 103(10), 1742.

[30] Dorronsoro, B., Danoy, G., \& Goire. (2013). Achieving super-linear performance in parallel multi-objective evolutionary algorithms by means of cooperative coevolution. Computers \& Operations Research, 40(6), 1552-1563.

[31] Malladi, S., Weaver, J. T., \& Clouse, T. L. (2011). Moving-average trigger for early detection of rapidly increasing mortality in caged table-egg layers. Avian Diseases, 55(4), 603-610.

[32] Rabiner, L., Sambur, M., \& Schmidt, C. Applications of a nonlinear smoothing algorithm to speech processing. IEEE Transactions on Acoustics Speech \& Signal Processing, 23(6), 552-557.

[33] Kalman, R. E. (1960). A new approach to linear filtering and prediction problems. Journal of Basic Engineering Transactions, 82, 35-45.

[34] Baraniuk, R. G. (1997). Improved wavelet de-noising via empirical wiener filtering. Proceedings of SPIE - The International Society for Optical Engineering, 3169, 389-399.

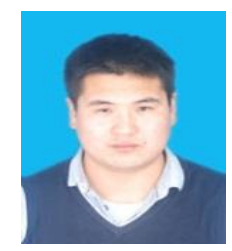

Jiang Zhipeng is a graduate student of Information Science and Engineering College of Shandong Normal University. Following Mr. Luo, he has been studying for more than two years, including data analysis, artificial intelligence and prediction of financial time series. The main interests are artificial intelligence and time series prediction.

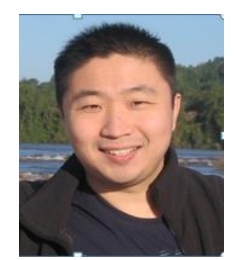

Luo Chao is mainly engaged in the theory and application research of evolutionary game, complex network and intelligent information processing of financial big data based on machine learning. 